\title{
$\beta$-Caryophyllene inhibits monoacylglycerol lipase activity and increases 2-AG levels: a new mechanism of endocannabinoid-mediated analgesia?
}

\author{
Jost Klawitter $^{1}$, Weibke Weissenborn ${ }^{1}$, MacKenzie Walz ${ }^{1}$, Jelena Klawitter ${ }^{1}$, Matthew \\ Jackson $^{1}$, Cristina Sempio ${ }^{1}$, Sonja Joksimovic ${ }^{1}$, Touraj Shokati ${ }^{1}$, Ingo Just ${ }^{2}$, Uwe \\ Christians $^{1}$, and Slobodan M. Todorovic ${ }^{1}$ \\ ${ }^{1}$ University of Colorado Denver School of Medicine \\ ${ }^{2}$ Hannover Medical School
}

July 4, 2021

\begin{abstract}
Introduction. $\beta$-Caryophyllene (BCP) has been shown to be an effective anti-inflammatory agent in chronic and inflammatory pain models. Since limited data are available for BCP in acute pain, we tested efficacy of BCP in an acute post-surgical pain model. Methods. BCP was tested in an acute postsurgical pain model. Animals were treated with vehicle, 10, 25, 50 and $75 \mathrm{mg} / \mathrm{kg} \mathrm{BCP}$ that was injected intra-peritoneally (i.p.). Time dependent paw withdrawal response (PWR) were evaluated using von Frey filaments and plasma and tissue samples were taken. BCP levels were determined in tissue (paw and spine) and plasma using an HPLC-MS based approach. Endocannabinoids (2-arachidonoylglycerol) were also evaluated in plasma and tissues using an HPLC-MS based approach. Monoacylglycerol lipase (MAGL) activity was evaluated in-vitro as well as ex vivo. Results. A dose-dependent improvement of hyperalgesia was observed up to $85 \%$ of the baseline value 30 minutes after administration of the highest BCP dose of $75 \mathrm{mg} / \mathrm{kg}$. A BCP dose-dependent increase in the 2-arachidonoylglycerol (2-AG) levels was observed with $9.9 \pm 6.4 \mathrm{ng} / \mathrm{mL}$ in the $75 \mathrm{mg} / \mathrm{kg}$ dose group as compared to vehicle controls with $3.0 \pm 2.5 \mathrm{ng} / \mathrm{mL}$. In vitro MAGL enzyme activity assessment using 2-AG as the substrate revealed an IC50 of $7.4 \mu \mathrm{M}$ of BCP for MAGL inhibition. Conclusion. These data showed that BCP inhibits MAGL activity in-vitro and in-vivo causing 2-AG levels to rise. Since the endocannabinoid 2-AG is a $\mathrm{CB} 1$ and $\mathrm{CB} 2$ receptor agonist, we propose the 2-AG-mediated cannabinoid receptor activation may contribute to BCP's mechanism of analgesia.
\end{abstract}

\section{Introduction}

Pain is a pervasive problem that affects millions of Americans each year. Over recent years the prescription of pain medications including opioids has skyrocketed [1]. However, surprisingly there has been very little progress or innovation in the field of chronic pain [2]. In the past 50 years the following four classes of drugs have been the mainstay of pain treatment: opioids, non-steroidal anti-inflammatory drugs, antidepressants (amitriptyline), antiepileptics (gabapentin), ziconitide, and ketamine; fewer than 10 medications with new mechanisms of action have become available, and only one now in clinical use was designed based on specific mechanisms of action (triptans for migraine) [2].

$\beta$-Caryophyllene ( $\mathrm{BCP}$, trans- $\beta$-Caryophyllene) is a major plant volatile compound found in large amounts in the essential oils of many different spice and food plants, especially clove oil, essential oils of Cannabis sativa, rosemary, and hops [3]. The Research Institute for Fragrance Materials (RIFM) evaluated BCP safety, and 
the molecule has been approved by the Food and Drug Administration and by the European Food Safety Authority as a flavoring agent, which can be used in cosmetic and food additives $[4,5]$. BCP has been reported to be a promising agent for treating several disorders, with particular reference to cancer, chronic pain and inflammation $[4,6]$. The efficiency of BCP in pain and inflammation and its well-established low toxicity make BCP an excellent drug candidate for the treatment of pain. BCP has been extensively studied for its antinociceptive and anti-inflammatory properties, however its mechanism of action remains largely unknown [7]. Direct interaction of BCP with the cannabinoid receptor CB2 has been postulated by Gertsch et. al [8]. Based on the possible binding of $\mathrm{BCP}$ to $\mathrm{CB} 2, \mathrm{BCP}$ was often described to belong to the cannabinoid family (binding to a cannabinoid receptor). Numerous investigators described BCP as a putative CB2 agonist based on this classification. However, this mechanism and resulting classification was recently disputed by other studies which controversially observed weak or no direct BCP binding to cannabinoid receptors [9-11].

In this study, we investigated the analgesic properties of BCP on surgically induced hyperalgesia using plantar incision of the hind paw in rats $[12,13]$, which is a rodent model considered to be similar in context to the underlying mechanisms and the development of human post-operative pain [14]. We show an alternative mechanism by which $\mathrm{BCP}$ alters the $\mathrm{CB} 2$ activity without the need to directly bind to the receptor. In this study we demonstrate that BCP inhibits monoacylglycerol lipase activity (MAGL) at pharmacologically relevant concentrations and consequently increases the endogenous levels of the CB2 and CB1 receptor agonist 2-arachidonoylglycerol (2-AG). The increase in levels of the endocannabinoid 2-AG and consequent activation of CB2 (and CB1) could contribute to the anti-hyperalgesic and anti-inflammatory properties of BCP.

\section{Methods}

Chemicals and reagents. Solvents and reagents (HPLC grade acetonitrile, methanol, water, the mobile phase constituent formic acid) used for mobile phases and sample extraction in this study were purchased from Fisher Scientific (Fair Lawn, NJ, USA) and used without further purification. The solvent (ethanol) used for the preparation of stock solutions and phosphate buffered saline (PBS0 was purchased from Sigma-Aldrich Chemicals (St. Louis, MO, USA). All study compound reference materials including anandamide (arachidonoyl ethanolamide, AEA), 2-arachionoylglycerol (2-AG), 1-arachionoylglycerol (1AG), docosatetraenoyl ethanolamide (DEA), dihomo- $\gamma$-linoleoylethanolamide (DH-g-LEA), N-arachidonoyl dopamine (NADA), 2-arachidonoylglycerol ether (2-AGE), O-arachidonoylethanolamide (O-AEA), N-oleoyl dopamine (ODA), oleamide (OLA), linoleoyl ethanolamide (LEA), oleoyl ethanolamine (OEA), palmitoyl ethanolamide (PEA) and stearoyl ethanolamide (SEA) were received from Cayman Chemical Company (Ann Arbor, MI, USA). All isotope labeled internal standards including 2-arachidonoylglycerol-d5 (2-AGd5), anandamide-d4 (AEA-d4), N-arachidonoyl dopamine-d8 (NADA-d8), linoleoyl ethanolamide-d4 (LEAd4), oleoyl Ethanolamide-d4 (OEA-d4), palmitoyl ethanolamide-d4 (PEA-d4) and stearoyl ethanolamide-d3 (SEA-d4) were also received from Cayman Chemical Company (Ann Arbor, MI, USA).

For the enrichment of QC samples blank human $\mathrm{K}_{2}$ EDTA plasma was from Bioreclamation IVT (Westbury, NY, USA) and charcoal stripped plasma used for calibrator standard preparation from BioCheMed (Winchester, VA, USA).

Animals. All in vivo experiments were performed with young female Sprague-Dawley rats (3-5 months, Envigo, Indianapolis, IN, USA). Animals were housed two per cage and maintained on a $12 \mathrm{~h}$ light-dark cycle with access to food and water ad libitum. Experimental protocols were in accordance with the Guide for the Care and Use of Laboratory Animals (Institute of Laboratory Animal Resources, 1996), and were approved by the Animal Care and Use Committee of the University of Colorado Anschutz Medical Campus (Aurora, CO, USA). We used female rats in our study for two main reasons: (1) females are considered to be more prone to chronic pain states when compared to males in both clinical and preclinical studies [15]; 
(2) the majority of current pain studies in animals are performed using males [15]. Thus, we believe that the research focus should also be on female pain perception $[13,16]$.

Incision pain model. In order to study the antihyperalgesic effect of BCP after surgery, we used a skin and muscle incision for the induction of post-surgical pain. Our standard model for the induction of postsurgical pain using the skin and muscle incision was described previously [13, 16-18] as follows: animals were anesthetized with isoflurane (2.5\% for induction and for maintenance) and the plantar surface of the right paw was incised longitudinally with a blade No.11; the underlying plantaris muscle was elevated and also longitudinally incised, whereafter the skin was sutured with 5-0 nylon suture with a FS-2 needle. Animals were allowed to recover in cages, and all experiments were initiated as early as $2 \mathrm{~h}$ post-incision.

Drug administration. BCP was dissolved in a solution of ethanol/ Cremophor/ $0.9 \%$ saline 1/1/18 in accordance with previous published protocols $[19,20]$. The tested doses were $10 \mathrm{mg} / \mathrm{kg}, 25 \mathrm{mg} / \mathrm{kg}, 50$ $\mathrm{mg} / \mathrm{kg}$ and $75 \mathrm{mg} / \mathrm{kg}$. The dissolved drug or vehicle solution was administered i.p. at the volume of $2 \mathrm{ml} / \mathrm{kg}$ (10 and $25 \mathrm{mg} / \mathrm{kg}$ group and 8 vehicle controls), $4 \mathrm{~mL} / \mathrm{kg}$ (50 mg/kg group and 4 vehicle controls) and 6 $\mathrm{mL} / \mathrm{kg}(75 \mathrm{mg} / \mathrm{kg}$ group and 4 vehicle controls). No volume administration based differences were observed within the vehicle group. Eight animals $(n=8)$ were randomly assigned to each dose group and 16 animals were in the vehicle control group.

Mechanical Sensitivity. Our standard method for assessing mechanical sensitivity was described previously $[16,17]$. Briefly: we used the electronic Von Frey apparatus (Ugo Basile, Varese, Italy), which utilizes a single rigid filament that exerts pressure to the plantar surface of the paw in a range from 0 to $50 \mathrm{~g}$. Animals were placed in plastic enclosures on a wire mesh stand to habituate for $15 \mathrm{~min}$. After habituation, a probe was applied to the plantar surface of the paw through the mesh floor of the stand, and constant force was applied to the mid-plantar area of the paw. As soon as the exerted pressure of the punctate stimulus reaches the maximum force that the animal can endure, immediate brisk paw withdrawal is noticeable, and the force in grams is displayed on the apparatus representing a threshold for paw withdrawal response (PWR). Each paw was tested three times and the average value of threshold PWRs was used in further analysis. Any other voluntary movement of the animal was not considered as a response.

Sample collection. After completion of the mechanical sensitivity testing animals received a second dose of $\mathrm{BCP}$ or vehicle solution. 30 minutes after this dose the animals were anesthetized using isoflurane and blood was drawn, spinal cord and paw tissue surrounding the incision site were collected.

Endocannabinoid analysis. Analysis of 14 endocannabinoids and congeners was performed in plasma, paw tissue and spinal cord tissue. A modification of a previously published assay was used [21]. The steps performed for the extraction of plasma and tissue are listed in detail in the supplementary methods section.

Monoacylglycerol lipase activity assay. A modification of a commercially available MAGL inhibitor screening kit (Item No. 705192, Cayman Chemical Company, Ann Arbor, MI) was used. Initial experiments showed interference of BCP with the colorimetric readout of the assay. Thus, the artificial substrate 4nitrophenylacetate at $236 \mu \mathrm{M}$ was substituted with the natural substrate 2-arachidonoylglycerol at the concentration of $236 \mu \mathrm{M}$. Consequently, the assay readout was arachidonic acid (AA) instead of 4-nitrophenol, which was measured using an HPLC-MS approach instead of a colorimetric readout. This principle was applied in-vitro andin-vivo .

In-vitro MAGL activity. After addition of $10 \mu \mathrm{L}$ MAGL, $10 \mu \mathrm{L}$ of ethanol solution or BCP solution in ethanol, and $10 \mu \mathrm{L}$ of 2-AG $(0.85 \mathrm{mM})$ to $150 \mu \mathrm{L}$ of the MAGL assay buffer (10 mM Tris-HCl, $\mathrm{pH} 7.2,1 \mathrm{mM}$ EDTA) supplied by the kit, the samples were incubated a room temperature for 20 minutes. The total volume of the reaction mixture was $180 \mu \mathrm{L}$. The reaction was terminated by addition of $800 \mu \mathrm{L}$ methanol including 1.25 $\mu \mathrm{g} / \mathrm{mL}$ of the internal standard arachidonic acid-d8 (Cayman Chemical Company, Ann Arbor, MI). Samples were centrifuged and supernatants were placed into HPLC vials for analysis. The high-performance liquid chromatography - tandem mass spectrometry (HPLC-MS/MS) parameters are listed in the Supplementary Materials and Methods section. 
In-vivo MAGL activity. For in-vivo MAGL activity assessment 25 to $41 \mathrm{mg}$ of spinal cord tissue were homogenized over liquid nitrogen using precooled mortar and pestle. The powder was added to $85 \mu \mathrm{L}$ of MAGL assay buffer in a tube on a scale and the added weight of tissue was recorded. Five $\mu \mathrm{L}$ of 2-AG-d8 $(0.85 \mathrm{mM})$ were added to the slurry and incubated for 60 minutes at $37^{\circ} \mathrm{C}$. The reaction was terminated by addition of $400 \mu \mathrm{L}$ methanol including $1.25 \mu \mathrm{g} / \mathrm{mL}$ of the internal standard arachidonic acid-d5 (Cayman Chemical Company, Ann Arbor, MI). Samples were centrifuged and supernatants were placed into HPLC vials for analysis. The high-performance liquid chromatography - mass spectrometry parameters are listed in the Supplementary Materials and Methods section.

$\beta$-Caryophyllene (BCP) analysis. Stock solutions of BCP were prepared in ethanol at $1 \mathrm{mg} / \mathrm{mL}$. This was used to generate calibration curves in the range between $0.0025 \mu \mathrm{g} / \mathrm{mL}$ to $10 \mu \mathrm{g} / \mathrm{mL}(0,0.0025,0.005$, $0.01,0.02,0.05,0.1,0.4,1,4,10 \mu \mathrm{g} / \mathrm{mL})$. For tissue analysis paw tissue and spine tissue were manually homogenized over liquid nitrogen using a mortar and pestle. Approximately $50 \mathrm{mg}$ of tissue powder were added to a pre-weighted microcentrifuge tube containing $500 \mu \mathrm{L}$ of acetonitrile and the actual tissue weight was noted. Samples were vortexed thoroughly Multitube vortexer (Fisher Scientific, Waltham, MA, USA) and centrifuged at $25,000 \mathrm{xg}$ and $4^{\circ} \mathrm{C}$ for $10 \mathrm{~min}$ (MR 23i centrifuge with a fiberlite rotor, Thermo Scientific, Waltham, MA, USA). Thirty $\mu \mathrm{L}$ of the supernatant was transferred into HPLC vials with conical inserts. To this $5 \mu \mathrm{L}$ of the internal standard $(1 \mu \mathrm{g} / \mathrm{mL}$ linalool $)$ solution was added and briefly vortexed. For plasma, 40 $\mu \mathrm{L}$ of sample was placed into a $1.5 \mathrm{~mL}$ micro centrifuge tube. Then $6 \mu \mathrm{L}$ of the internal standard $(1 \mu \mathrm{g} / \mathrm{mL}$ linalool) solution was added. Samples were mixed briefly and $160 \mu \mathrm{L}$ of acetonitrile was added to each sample, were vortexed thoroughly on a multitube vortexer (Fisher Scientific, Waltham, MA, USA) and centrifuged at 25,000 $\mathrm{xg}$ and $4^{\circ} \mathrm{C}$ for $10 \mathrm{~min}$ (MR 23i centrifuge with a fiberlite rotor, Thermo Scientific, Waltham, MA, USA). One hundred $\mu \mathrm{L}$ of the supernatant was transferred into an HPLC vial with insert. Samples were analyzed using an Agilent 1200 series HPLC interfaced with an ABSciex API5000 MS/MS system (Sciex, Foster City, CA, USA) via an IonDrive Turbo V Source operated in positive atmospheric pressure ionization (APCI) mode. Ten $\mu \mathrm{L}$ of extract were injected onto a Halo C8 analytical column $(2.7 \mu \mathrm{m}, 3.0 x 100 \mathrm{~mm}$, MACMOD Analytical Inc. Chadds Ford, PA) and a gradient using $0.1 \%$ formic acid in water as aqueous buffer and methanol with $0.1 \%$ formic acid $(1 / 1, \mathrm{v} / \mathrm{v})$ as organic buffer was applied to achieve the chromatographic separation of BCP and linalool from endogenous compounds. The tandem mass spectrometry detector was operated in positive multiple reaction monitoring (MRM) mode. The electron potential was set to $-10 \mathrm{eV}$ and the collision cell exit potential to $-12 \mathrm{eV}$. The mass spectrometer was set to acquire $\mathrm{BCP}$ and the internal standard linalool (m/z, parent>daughter ion): 205>149, 205>95 and 205>55 for BCP; $137>81$ for the internal standard linalool.

Data Analysis. Once the data were acquired, MultiQuant, OS-MQ version 1.7 (Sciex) software was used for initial data processing and plasma level determination of lipids and endocannabinoids. Calibration curves were prepared in surrogate buffer. Both assays were validated as considered fit for purpose following generally applicable biomarker validation guidelines with accuracy and precision acceptance criteria of 80-120\% and $<20 \%$-CV, respectively. Resulting metabolite concentrations were normalized to the tissue weight and used for all subsequent statistical analyses.

Statistical data analysis. For statistical analysis IBM SPSS software version 27 (IBM, Armonk, NY, USA) was used. Data were analyzed without previous log-transformation. Distribution statistics included the calculation of means, standard deviations and standard errors of the mean (SEM). Groups were compared using analysis of variance (ANOVA) in combination with Tukey's post-hoc test.

\section{Results}

To assess the analgesic properties of $\mathrm{BCP}$ and its potential mechanism of action, we focused on the postsurgical incision pain model. The effects of $\mathrm{BCP}$ on mechanical hyper-analgesic effects in rats after paw surgery when administered in escalating doses at 10, 25, 50 and $75 \mathrm{mg} / \mathrm{kg}$ i.p. were compared to the vehicle administration. In animals that underwent skin incision, we focused on mechanical hyper-analgesic effects as an 
important feature of incisional pain for two main reasons: it is very commonly observed in a clinical setting of incisional pain $[16,22,23]$ and, as published previously, it is sensitive to pharmacological and biochemical modulation $[16,17]$. The paw withdrawal responses (PWR) were determined in the incised (hyper analgesic effect) right hind paw (Figure 1 left side) and non-operated (left) paws (Figure 1 right panels). We noticed that BCP caused a dose-dependent increase of the PWRs in operated paws indicating a selective analgesic effect. The baseline PWR for the right paws prior to the incision was $38.5 \pm 4.5$ grams (mean \pm standard deviation). At 2 hours post-surgery this value dropped to $9.7 \pm 4.5$ grams indicating hyper analgesic effects. While the vehicle group $(\mathrm{n}=16)$ remained at an average of $10.5 \pm 2.0$ grams during the treatment period, all BCP treated animals showed diminished paw withdrawal responses at 15- and 30-minutes post injection. These changes were most pronounced for the $75 \mathrm{mg} / \mathrm{kg}$ BCP treatment group $(\mathrm{n}=8)$ showing PWR improvements with $31.4 \pm 4.1$ and $32.9 \pm 3.5$ grams for 30 and 60 minutes post injection, respectively. The paw withdrawal responses for this group were only $15 \%$ lower than the pre-surgery values $(38.5 \pm 4.5$ grams). The PWR were also evaluated in the non-incised left hind-paws of study animals (Figure 1 right side). On the day of the PWR assessments the left paws showed an average of $42.0 \pm 4.0$ grams for the vehicle group. Except for a moderate and transient increase in PWR in the $75 \mathrm{mg} / \mathrm{kg}$ group at 15 minutes post i.p. injection $(51.7 \pm 6.6$ grams), there were no significant changes in the left paw PWR amongst all treatment group compared to the vehicle control. This indicates that BCP is a relatively selective analgesic for affected paws following surgical skin incision.

Next, to further gain insight into the mechanism of BCP analgesic activity, we evaluated changes in endocannabinoid plasma and tissue concentrations using a comprehensive endocannabinoid analysis including 14 endocannabinoids and congeners in plasma and tissue samples. We noticed that plasma 2-AG concentrations increased in parallel with increasing BCP administration (Figure 2). Amongst the 14 endocannabinoids analyzed we observed statistically significantly increased 2-AG levels in plasma in the $75 \mathrm{mg} / \mathrm{kg}$ BCP dose group about 3-fold. Mean 2-AG plasma levels for the vehicle control group were $3.0 \pm 2.6 \mathrm{ng} / \mathrm{mL}$. With the exception of the $10 \mathrm{mg} / \mathrm{kg}$ group all other treatment groups showed increased 2-AG plasma levels with the highest value for the $75 \mathrm{mg} / \mathrm{kg}$ group with $9.9 \pm 6.4 \mathrm{ng} / \mathrm{mL}(\mathrm{p}<0.001)$. Absolute tissue concentrations of 2-AG in paw tissue and spinal cord tissue resulted in no significant changes between study groups. However, when a potential association between spinal cord tissue 2-AG and BCP concentrations was assessed (Pearson Correlation), a significant correlation $(\mathrm{p}=0.0024)$ with a correlation coefficient $\mathrm{r}=0.518$ was observed (supplementary figure $\mathrm{S} 2$ ). No such correlation was observed in paw tissues.

To test our hypothesis that the increase of 2-AG concentrations was caused by an effect of BCP on monoacylglycerol lipase (MAGL) activity, a commercially available MAGL inhibitor screening assay kit (Cayman Chemicals) was initially tested (Figure 3A). For this application, the assay showed interference of BCP with the colorimetric readout (Figure 3A). Thus, a modification of the assay was developed in which the natural MAGL substrate 2-arachidonoylglycerol (2-AG) was used and the product, arachidonic acid (AA), was quantified using HPLC-MS/MS (see supplementary Figure S1). After optimization of assay parameters (see methods) the assay was applied on consecutive 4 days with 2 assessments per day. Under these conditions the $\mathrm{IC}_{50}$ for $\mathrm{BCP}$ was determined to be $7.4 \mu \mathrm{M}$ (95\% confidence interval 3.9 to 14.0). The correlation coefficient for the curve fit was $\mathrm{r}=0.9445$.

Monoacylglycerol lipase activity was also assessed in spinal cord tissues. Animals were treated with BCP at $0 \mathrm{mg} / \mathrm{kg}$ (vehicle controls), as well as 10, 25, 50 and $75 \mathrm{mg} / \mathrm{kg}$. Tissue was harvested 30 minutes after BCP administration, frozen in liquid nitrogen and stored at $-70^{\circ} \mathrm{C}$ or below until analysis. Due to the natural abundance of 2-AG and arachidonic acid in tissue, samples were placed into a slurry with MAGL activity assay buffer and incubated with labeled 2-AG-d8. After the incubation period, the tissue was extracted, and the AA-d8 content was analyzed (for details see methods section and supplementary Figure S1). Formation of AA-d8 was reduced in spinal cord tissues of animals previously treated with BCP. Vehicle controls showed an AA-d8 formation rate of $3.76 \pm 0.96 \mathrm{pg} \cdot \mathrm{mg}^{-1} \cdot \mathrm{min}^{-1}$. The values for the treatment groups were $3.56 \pm 1.01$ $\mathrm{pg} \cdot \mathrm{mg}^{-1} \cdot \mathrm{min}^{-1}, 3.16 \pm 0.57 \mathrm{pg} \cdot \mathrm{mg}^{-1} \cdot \mathrm{min}^{-1}, 2.99 \pm 1.12$ and $2.58 \pm 0.73(\mathrm{p}=0.036)$ for the $10,25,50$ and 75 $\mathrm{mg} / \mathrm{kg}$ treatment groups, respectively. 
BCP drug levels were determined in plasma, spinal cord and paw tissue 30 minutes after a second administration of BCP to estimate plasma and tissue levels and correlate in-vitro data to in-vivo levels. A high-performance liquid chromatography (HPLC) atmospheric pressure chemical ionization (APCI) multiple reaction monitoring (MRM) tandem mass spectrometry (MS/MS) assay was developed to quantify BCP with high specificity and selectivity. Representative extracted ion chromatograms of BCP in plasma samples is shown in Supplementary Figure S3. The assay was linear from 0.49 to $490 \mathrm{nmol}$. All plasma and tissue samples of treated animals were within the linear range of the assay. Tissue levels were normalized to the amount of tissue extracted with acetonitrile and are presented as nmol/g of tissue to allow for easy comparison to plasma level, which are presented in $\mathrm{nmol} / \mathrm{mL}$ (see Figure $5 \mathrm{~A}-5 \mathrm{C}$ ). BCP plasma levels were 2.34 $\pm 1.34,4.60 \pm 2.33,5.48 \pm 1.88$ and $15.90 \pm 4.84 \mathrm{nmol} / \mathrm{mL}$ (mean \pm standard deviation) for the 10, 25, 50 and $75 \mathrm{mg} / \mathrm{kg}$ treatment groups, respectively. Paw tissue levels were higher than plasma levels with 6.16 $\pm 2.22,9.73 \pm 3.56,14.68 \pm 6.38$ and $40.37 \pm 10.62 \mathrm{nmol} / \mathrm{g}$ for the $10,25,50$ and $75 \mathrm{mg} / \mathrm{kg}$ treatment groups, respectively. Interestingly, the spinal cord concentrations were even 8 to 9 -fold higher in comparison to plasma and 3 to 4 -fold higher in comparison to paw tissue depending on the treatment group. Spinal cord BCP concentrations reached $18.61 \pm 6.94,39.13 \pm 13.78,50.38 \pm 25.09,133.85 \pm 22.92 \mathrm{nmol} / \mathrm{g}$ for the 10 , 25,50 and $75 \mathrm{mg} / \mathrm{kg}$ treatment groups, respectively (Figure $5 \mathrm{~A}-5 \mathrm{C}$ ).

\section{Discussion}

Following the findings of Gertsch et al. [8] BCP has been considered a putative CB2 agonist. However, although there is a lot of evidence from Gertsch et al. and others [8, 24-27], it remains controversial, whether $\mathrm{BCP}$ binds directly to $\mathrm{CB} 2$ and acts as an agonist or if the interaction is rather weak [9-11]. In this study we showed that $\beta$-caryophyllene inhibits monoacylglycerol lipase at pharmacologically relevant concentrations and consequently increased the levels of the Cannabinoid receptor 2 (CB2) agonist 2-arachidonoylglycerol. Hence, the inhibition of monoacylglycerol lipase may contribute to the putative CB2 agonism of BCP. Importantly, many characteristic properties of $\mathrm{BCP}$, such as the anti-hyperalgesic and anti-inflammatory properties, could be explained via this mechanism of action. However, the proposed mechanism - inhibition of MAGL - results in abolishing the selectivity of BCP to CB2- receptors as 2-AG acts agonistically to both CB1- and CB2-receptors.

$\beta$-Caryophyllene is approved by the United States Food and Drug Administration and European agencies as a food additive, taste enhancer, and flavoring agent [28]. It also displays important pharmacological activities such as anticancer, cardioprotective, hepatoprotective; gastroprotective, nephroprotective, antioxidant, antiinflammatory, antimicrobial, immune-modulatory, and neuroprotective [7, 28-30]. $\mathrm{BCP}$ has the potential to treat several neurological diseases and disorders such as cerebral ischemia, brain lesions, neuro-inflammation, and problems in cortical, hippocampal, and cerebellar neurons and glial cells [7,31-35]. The analgesic effect of BCP in essential oils and other formulations has been well described [19, 36-41]. BCP rich essential oils have demonstrated antinociceptive effects at $25-62.5 \mathrm{mg} / \mathrm{kg}$ [40], $50-75 \mathrm{mg} / \mathrm{kg}$ [37], and $100-400 \mathrm{mg} / \mathrm{kg}$ $[36,39]$ (p.o.). However, BCP content in essential oils is often variable and surprisingly, pure BCP displays similar analgesic activities as several essential oils, in which BCP is a major active compound [29]. This might be related to bioavailability and contribution of other essential oil components to the effect of BCP. Pure $\beta$-caryophyllene has been tested at various concentrations in single and multiple administration dose designs. In this context BCP showed to be antinociceptive at $1-5 \mathrm{mg} / \mathrm{kg}$ in mice [19], $2.6 \mathrm{mg} / \mathrm{kg} / \mathrm{day}$ for 2 weeks in mice [42] and $25 \mathrm{mg} / \mathrm{kg}$ [41]. Most of these models tested the antinociceptive effect of BCP in formalin, acetic acid, and tail immersion tests. To our knowledge the anti-hyperalgesic effects of BCP in an acute post-surgical pain model has not been previously studied. We have observed a BCP dose dependent increase of the PWR preferentially in operated paws using this clinically relevant model of post-operative pain (Figure 1). However, we cannot exclude an additional anti-inflammatory effect contributing to the analgesic effect. The lowest BCP dose tested $(10 \mathrm{mg} / \mathrm{kg})$ showing significantly higher PWRs $(145-167 \%)$ at 15,30, 60 and 90 minutes after injection compared to vehicle controls (100\%). Administration of 75 $\mathrm{mg} / \mathrm{kg}$ resulted in paw withdrawal responses at 30 minutes post injection $(32.9 \pm 3.5$ grams $)$ that were 
$85 \%$ of the mean PWR for all baseline values prior to the incision (38.5 \pm 4.5 grams) indicating a potent anti-hyperalgesic effect of BCP. These data show a markedly postoperative analgesic effect of BCP while non-operated paws were only slightly affected with the highest dose of BCP. The difference to the effective doses in the mice formalin/acetic acid and tail immersion tests is likely based on the characteristics of the different pain models.

Key components of the endocannabinoid system are expressed throughout nociceptive pathways: in the periphery on primary afferent neurons, in the dorsal horn of the spinal cord and in multiple supraspinal regions of the brain associated with pain perception and modulation [43]. As a result, targeting the endocannabinoid system via enhancement of the levels of endogenous cannabinoids (e.g. with fatty acid amide hydrolase (FAAH) or MAGL inhibitors) or exogenous cannabinoid ligands (e.g. CB1 or CB2 receptor agonists) can reduce nociceptive transmission at all three of these neurophysiological levels [43]. The effects of BCP treatment on the endocannabinoid system was evaluated and changes in circulating endocannabinoids, specifically 2-AG were observed (Figure 2). These results led us to the hypothesize that BCP increases 2-AG levels via inhibition of MAGL. To test this hypothesis a commercially available MAGL inhibitor screening assay was used to evaluate BCP's ability to inhibit MAGL activity. However, due to interference of BCP with the colorimetric readout of the assay kit (Figure 3A) a modified strategy was applied using the natural substrate, 2-AG, for the MAGL activity assessment and analyzing the catalytic product arachidonic acid (AA) by HPLC-MS. A similar methodology has been applied by others $[44,45]$ mostly utilizing 2-oleoylglycerol (2-OG) as the substrate.

The $\mathrm{IC}_{50}$ for $\mathrm{BCP}$ was determined to be $7.4 \mu \mathrm{M}$. A similar approach was applied to test the MAGL activity in tissue homogenates of animals from the BCP treatment groups. For this deuterated 2-AG (2-AG-d8, Supplementary Figure S1) was used as the MAGL substrate. After incubation of the tissue slurry samples were extracted and the MAGL product AA-d8 was determined using HPLC-MS. A statistically significant decrease of AA-d8 formation was observed in spinal cord tissue from $75 \mathrm{mg} / \mathrm{kg}$ treated animals indicating a decreased hydrolysis of 2-AG-d8 in this treatment group (Figure 4). These data demonstrate that BCP inhibits MAGL in-vitro and in-vivo and most likely explains why BCP increased 2-AG levels in plasma and spinal cord tissue (supplemental Figure S2) in a dose dependent manner. It is well established that MAGL inhibition and increased 2-AG levels have an antinociceptive and anti-inflammatory effects [46-49]. Importantly, 2-AG is a CB1 and a CB2 agonist [50]. Therefore, we posit that the BCP-induced increased levels of 2-AG could contribute to the observed antinociceptive and well describe anti-inflammatory effects or BCP. The IC50 determined in the in-vitro MAGL inhibitor assay might not be identical to that of tissue MAGL. However, the BCP concentrations found in tissue (paw and spine) are much higher than the in-vitro IC50 value, depending on the treatment group. Also labeled 2-AG incubations of tissue ex vivo also indicates MAGL inhibition.

To determine if BCP's $\mathrm{IC}_{50}$ of $7.4 \mu \mathrm{M}$ can be reached in-vivo after BCP administration plasma, paw tissue and spinal cord tissue BCP concentrations have been determined by high performance liquid chromatography tandem mass spectrometry. Due to the different routes of administration and formulations of BCP, which is a lipophilic and volatile terpene, it is essential to determine plasma tissue levels to accurately interpret in-vitro and in-vivo experiments as well as allowing for a comparison of data gained from this study to other studies. Plasma BCP levels 30 minutes after a second administration did show a concentration dependent increase amongst the study groups (Figure 5). However, the increase is not linear for the 10, 25, 50 and $75 \mathrm{mg} / \mathrm{kg}$ treatment groups with mean values of $2.34,4.60,5.48,15.90 \mathrm{nmol} / \mathrm{mL}(\mu \mathrm{M})$, respectively. This non-linear level increase was reflected in paw and spinal cord tissue levels as well. BCP levels were increased in paw tissue and even further accumulated in spinal cord as compared to plasma, which can be explained by the very lipophilic nature of BCP. The concentrations of BCP observed are generally in alignment with the literature [51, 52]. Varga et al. determined 30 minutes after single dose administration of $10 \mathrm{mg} / \mathrm{kg}$ BCP i.p. in mice serum levels of ca. $1.2 \mathrm{nmol} / \mathrm{mL}$ [51] compared to $2.3 \mathrm{nmol} / \mathrm{mL}$ observed in rat plasma in this study after 30 minutes after the second BCP dose. Others performed pharmacokinetic analysis after oral administration of $50 \mathrm{mg} / \mathrm{kg} \mathrm{BCP}$ in rats [52,53] and determined $\mathrm{C}_{\max }$ levels $2.5-10 \mathrm{nmol} / \mathrm{mL}$ depending 1.5-3 hours after administration depending on the vehicle solutions used. In the present study 
we observed average plasma levels of $5.5 \mathrm{nmol} / \mathrm{mL}(\mu \mathrm{M})$ in the $50 \mathrm{mg} / \mathrm{kg}$ group. It is known from very lipophilic drugs administered by i.p. injection to result in very high CNS concentrations due to excellent blood supply in combination of high lipophilicity of the brain/spine. Afterwards redistribution takes place resulting in lowering of brain/spine concentrations and an increase in plasma concentrations. In this model we are not at a steady state concentration. Thus, a comparison of the kinetics of twice i.p. injections with oral administration is not possible.

It has been proposed that $\mathrm{BCP}$ is a putative $\mathrm{CB} 2$ receptor agonist [8, 24-27]. However, recently it was disputed whether BCP binds directly to $\mathrm{CB} 2$ and acts as an agonist or if the interaction is rather weak [9-11]. Alternatively, our data strongly suggest that MAGL inhibition and, consequently, increased levels of 2-AG, a known analgesic, at least contribute to the antinociceptive and anti-inflammatory effects observed with BCP administration. Administration of CB1 or CB2 agonists results in activation of all receptors in the body, and depending on tissue receptor density, might results in increased side effects. However, the modulation of local 2-AG concentrations by MAGL inhibition might be a more selective and targeted approach.

\section{Figure Legends}

\section{Figure 1}

Paw withdrawal response (PWR) observed using von Frey filament testing. Left side, PWR for the incised right paw for the vehicle control study group (black) and for the study groups with $\beta$-caryophyllene (BCP) treatment groups at 10, 25, 50 and $75 \mathrm{mg} / \mathrm{kg}$. Right side, the corresponding PWR for the non-incised left paws of the same study groups. Data are shown as mean \pm standard deviation. For the incised paw at 15, 30, 60 and 90 minutes after $\mathrm{BCP}$ administration, all treatment groups showed statistically significantly higher $(\mathrm{p}<0.01)$ mean PWRs compared to the vehicle control group.

\section{Figure 2}

Determination of 2-AG plasma levels. 2-AG levels were determined as part of a HPLC-MS/MS based endocannabinoid multiplex assay. ${ }^{* * *}$ indicates statistically significantly $(\mathrm{p}<0.001)$ changed compared to the vehicle control group based on post-hoc Tukey HSD test.

\section{Figure 3}

Determination of MAGL inhibition. (A) MAGL inhibitor activity evaluation using the commercially available MAGL inhibitor screening assay kit. The activity evaluation is based on the ability of MAGL to hydrolyze 4-nitrophenylacetate to form acetate and 4-nitrophenol, which has a specific extinction coefficient of 18,000 $\mathrm{M}^{-1} \mathrm{~cm}^{-1}$ at $405 \mathrm{~nm}$. The dashed area indicates interference of BCP at high concentrations with the colorimetric readout. (B) MAGL inhibition by BCP based on the natural substrate 2-AG, which is hydrolyzed by MAGL to glycerol and arachidonic acid (AA). AA concentrations were determined using HPLC-MS/MS. Data are shown as mean \pm standard error of the mean (SEM).

\section{Figure 4}

Determination of MAGL activity in spinal cord tissue. MAGL activity evaluation was performed using the deuterated substrate 2-AG-d8, which is hydrolyzed by MAGL to glycerol and AA-d8. AA-d8 concentrations were determined using HPLC-MS/MS using AA-d5 as internal standard. Data are shown as mean \pm SEM. * indicates statistically significantly $(\mathrm{p}<0.05)$ changed compared to the vehicle control group based on post-hoc Tukey's HSD test.

\section{Figure 5}

$\mathrm{BCP}$ concentrations in plasma and tissue 30 minutes after second $\mathrm{BCP}$ administration. BCP concentrations in plasma (A), paw tissue (B) and spinal cord tissue (C) are shown. Data are shown as mean + standard 
deviation. All data are $\mathrm{n}=8$, with the exception of the plasma levels for the $50 \mathrm{mg} / \mathrm{kg}$ group $(\mathrm{n}=6)$, for which 2 plasma samples were not available because of technical difficulties.

\section{Figures}

Figure 1

\section{Incised paws}

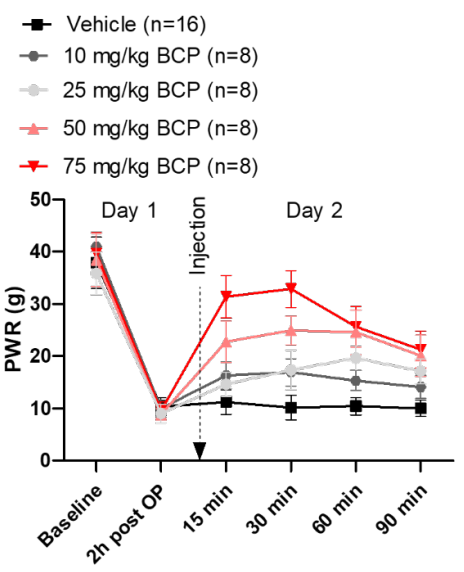

\section{Unincised paws}
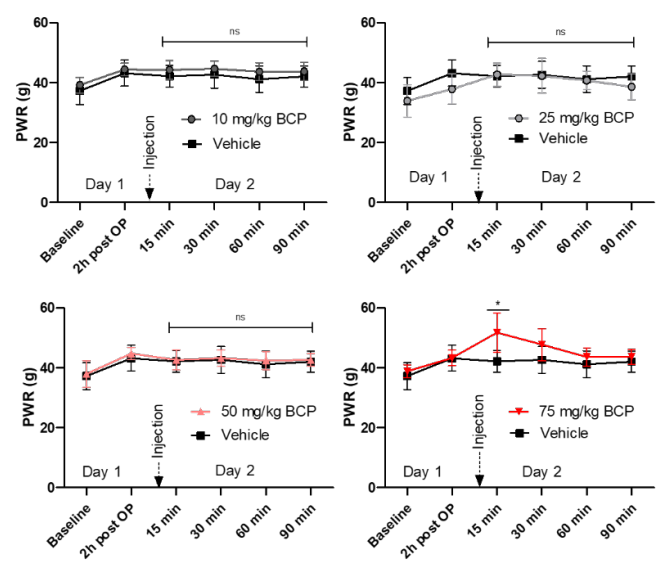

Figure 2

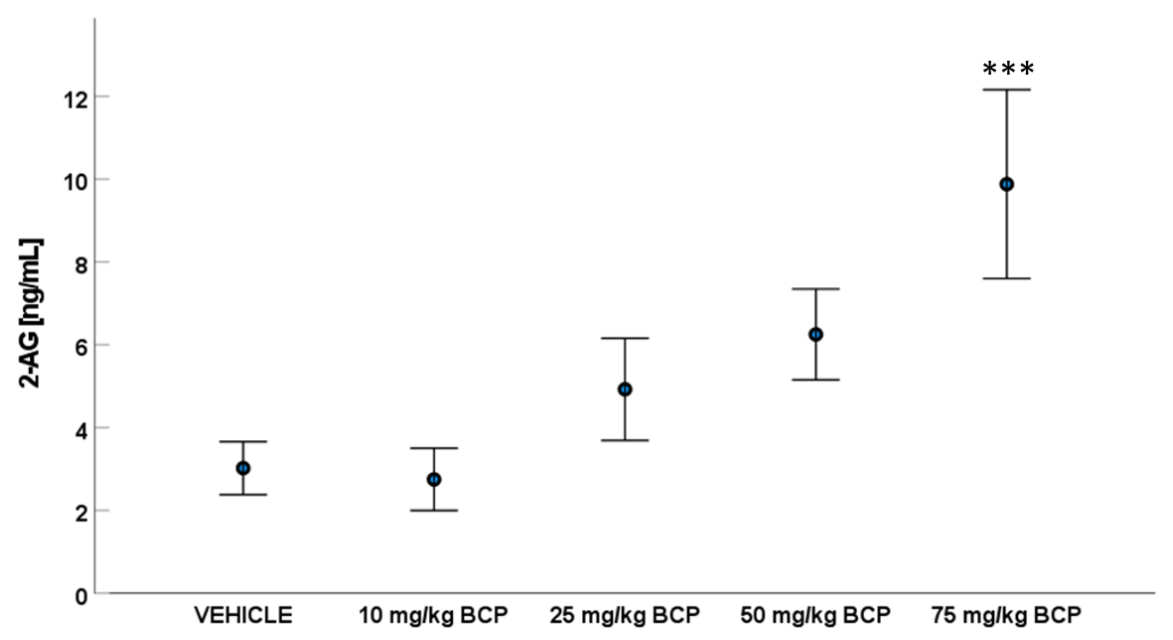

Figure 3 

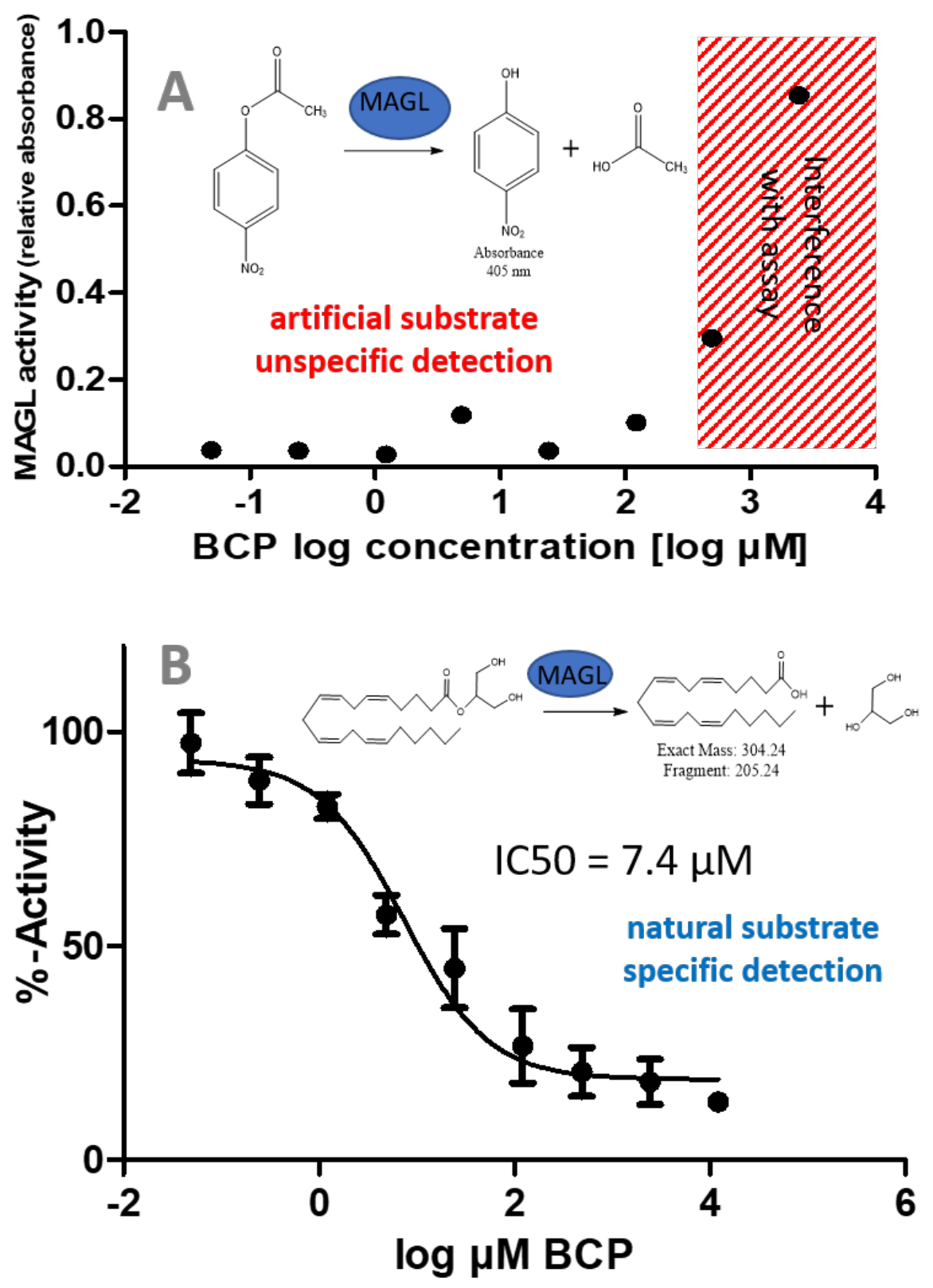

Figure 4 


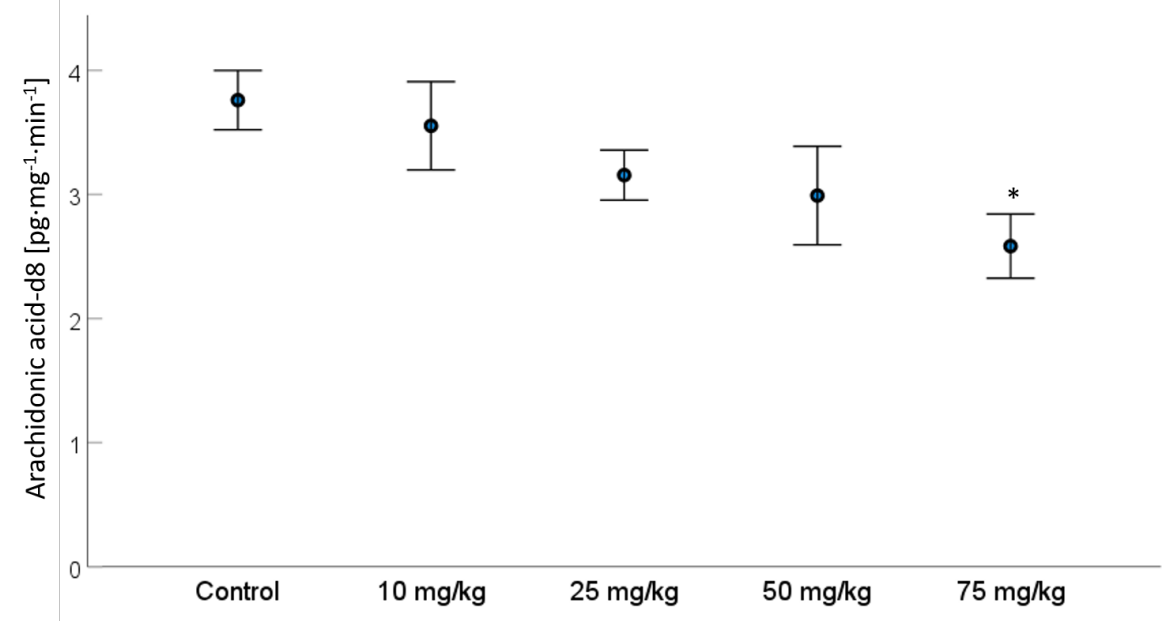

Figure 5 

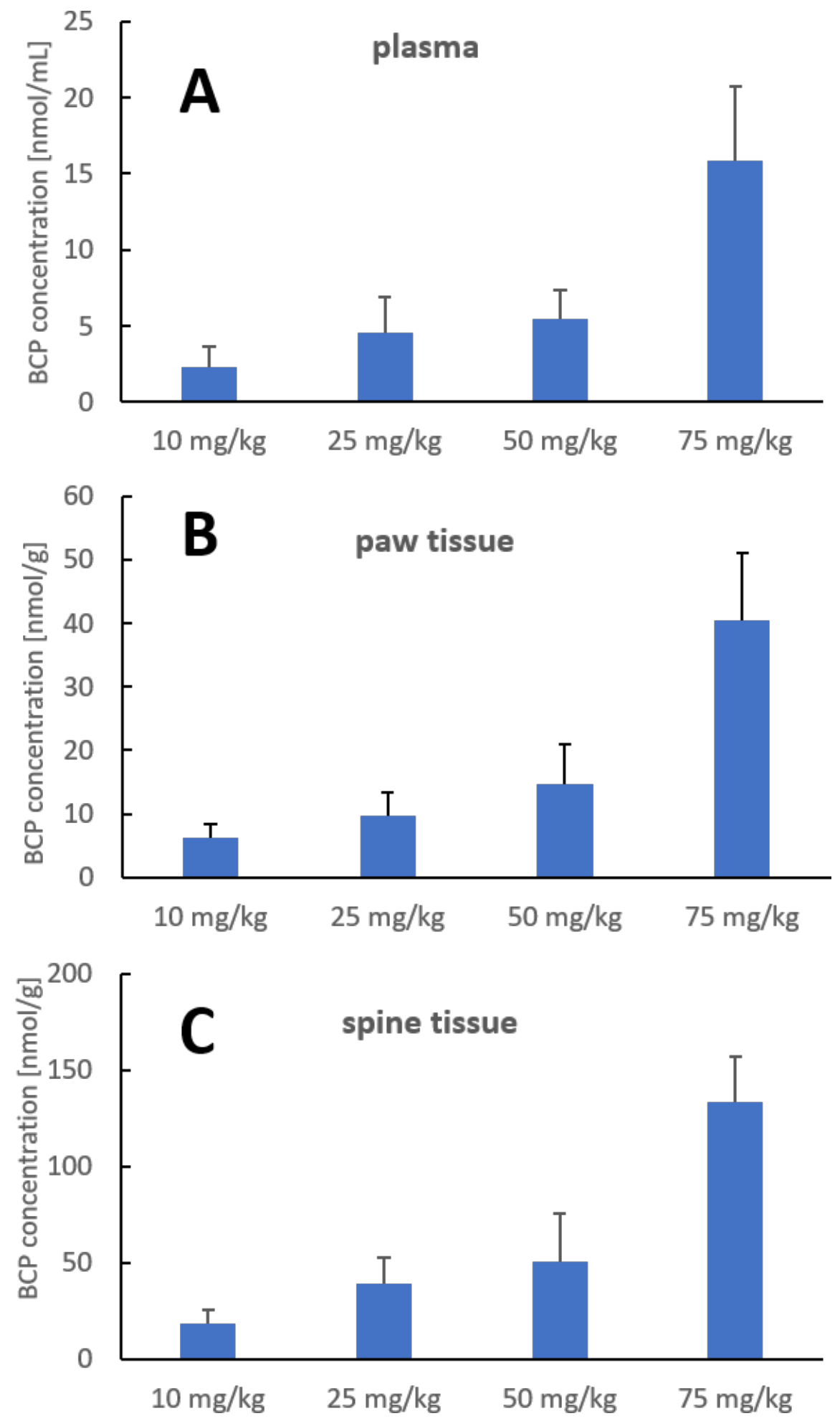


\section{References (ideally no more than 60, no specific format)}

1. Trecki, J., A Perspective Regarding the Current State of the Opioid Epidemic. JAMA Netw Open, 2019. 2 (1): p. e187104.

2. Borsook, D., A future without chronic pain: neuroscience and clinical research. Cerebrum, 2012. 2012 : p. 7 .

3. Ghelardini, C., et al., Local anaesthetic activity of beta-caryophyllene. Farmaco, 2001. 56 (5-7): p. 387-9.

4. Maffei, M.E., Plant Natural Sources of the Endocannabinoid (E)-beta-Caryophyllene: A Systematic Quantitative Analysis of Published Literature. Int J Mol Sci, 2020. 21 (18).

5. Schmitt, D., R. Levy, and B. Carroll, Toxicological Evaluation of beta-Caryophyllene Oil: Subchronic Toxicity in Rats. Int J Toxicol, 2016. 35 (5): p. 558-67.

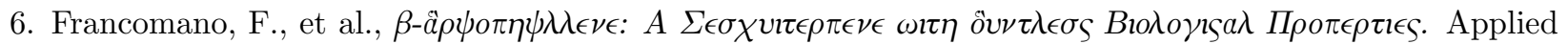
Sciences, 2019.9 (24): p. 5420.

7. Machado, K.D.C., et al., A systematic review on the neuroprotective perspectives of beta-caryophyllene. Phytother Res, 2018. 32 (12): p. 2376-2388.

8. Gertsch, J., et al., Beta-caryophyllene is a dietary cannabinoid. Proc Natl Acad Sci U S A, 2008. 105 (26): p. 9099-104.

9. Finlay, D.B., et al., Terpenoids From Cannabis Do Not Mediate an Entourage Effect by Acting at Cannabinoid Receptors. Front Pharmacol, 2020. 11 : p. 359.

10. Santiago, M., et al., Absence of Entourage: Terpenoids Commonly Found in Cannabis sativa Do Not Modulate the Functional Activity of Delta(9)-THC at Human CB1 and CB2 Receptors. Cannabis Cannabinoid Res, 2019. 4 (3): p. 165-176.

11. Heblinski, M., et al., Terpenoids Commonly Found in Cannabis sativa Do Not Modulate the Actions of Phytocannabinoids or Endocannabinoids on TRPA1 and TRPV1 Channels. Cannabis Cannabinoid Res, 2020. 5 (4): p. 305-317.

12. Brennan, T.J., E.P. Vandermeulen, and G.F. Gebhart, Characterization of a rat model of incisional pain. Pain, 1996.64 (3): p. 493-502.

13. Joksimovic, S.L., et al., Inhibition of multiple voltage-gated calcium channels may contribute to spinally mediated analgesia by epipregnanolone in a rat model of surgical paw incision. Channels (Austin), 2019. 13 (1): p. 48-61.

14. Pogatzki-Zahn, E.M., D. Segelcke, and S.A. Schug,Postoperative pain-from mechanisms to treatment. Pain Rep, 2017.2 (2): p. e588.

15. Mogil, J.S. and M.L. Chanda, The case for the inclusion of female subjects in basic science studies of pain. Pain, 2005.117 (1-2): p. 1-5.

16. Tat, Q.L., et al., Preemptive Analgesic Effect of Intrathecal Applications of Neuroactive Steroids in a Rodent Model of Post-Surgical Pain: Evidence for the Role of T-Type Calcium Channels. Cells, 2020.9 (12).

17. Joksimovic, S.L., et al., Novel neuroactive steroid with hypnotic and T-type calcium channel blocking properties exerts effective analgesia in a rodent model of post-surgical pain. Br J Pharmacol, 2020. 177 (8): p. $1735-1753$.

18. Joksimovic, S.L., et al., Selective inhibition of CaV3.2 channels reverses hyperexcitability of peripheral nociceptors and alleviates postsurgical pain. Sci Signal, 2018. 11 (545). 
19. Klauke, A.L., et al., The cannabinoid CB(2) receptor-selective phytocannabinoid beta-caryophyllene exerts analgesic effects in mouse models of inflammatory and neuropathic pain. Eur Neuropsychopharmacol, 2014. 24 (4): p. 608-20.

20. Yokubaitis, C.G., et al., Effects of Cannabidiol and Beta-Caryophyllene Alone or in Combination in a Mouse Model of Permanent Ischemia. Int. J. Mol. Sci., 2021. 22 (2866): p. 1-11.

21. Sempio, C., et al., Analysis of 14 endocannabinoids and endocannabinoid congeners in human plasma using column switching high-performance atmospheric pressure chemical ionization liquid chromatographymass spectrometry. Anal Bioanal Chem, 2021.413 (12): p. 3381-3392.

22. Zahn, P.K. and T.J. Brennan, Primary and secondary hyperalgesia in a rat model for human postoperative pain.Anesthesiology, 1999. 90 (3): p. 863-72.

23. Brennan, T.J., Pathophysiology of postoperative pain. Pain, 2011. 152 (3 Suppl): p. S33-S40.

24. Alberti, T.B., D.S. Coelho, and M. Maraschin,beta-Caryophyllene nanoparticles design and development: Controlled drug delivery of cannabinoid CB2 agonist as a strategic tool towards neurodegeneration. Mater Sci Eng C Mater Biol Appl, 2021.121 : p. 111824.

25. Mensching, L., et al., Monitoring Cannabinoid CB2 -Receptor Mediated cAMP Dynamics by FRETBased Live Cell Imaging. Int J Mol Sci, 2020. 21 (21).

26. Irrera, N., et al., beta-Caryophyllene Inhibits Cell Proliferation through a Direct Modulation of CB2 Receptors in Glioblastoma Cells. Cancers (Basel), 2020. 12 (4).

27. Irrera, N., et al., beta-Caryophyllene Mitigates Collagen Antibody Induced Arthritis (CAIA) in Mice Through a Cross-Talk between CB2 and PPAR-gamma Receptors. Biomolecules, 2019. 9 (8).

28. Chicca, A., et al., Functionalization of beta-caryophyllene generates novel polypharmacology in the endocannabinoid system. ACS Chem Biol, 2014. 9 (7): p. 1499-507.

29. Fidyt, K., et al., beta-caryophyllene and beta-caryophyllene oxide-natural compounds of anticancer and analgesic properties. Cancer Med, 2016. 5 (10): p. 3007-3017.

30. Sharma, C., et al., Polypharmacological Properties and Therapeutic Potential of beta-Caryophyllene: A Dietary Phytocannabinoid of Pharmaceutical Promise. Curr Pharm Des, 2016. 22 (21): p. 3237-64.

31. Zhang, M., et al., Modulation of the balance between cannabinoid CB(1) and CB(2) receptor activation during cerebral ischemic/reperfusion injury. Neuroscience, 2008. 152 (3): p. 753-60.

32. Zarruk, J.G., et al., Cannabinoid type 2 receptor activation downregulates stroke-induced classic and alternative brain macrophage/microglial activation concomitant to neuroprotection.Stroke, 2012. 43 (1): p. 211-9.

33. Zhang, M., et al., CB2 receptor activation attenuates microcirculatory dysfunction during cerebral ischemic/reperfusion injury. Microvasc Res, 2009. 78 (1): p. 86-94.

34. Pertwee, R.G., Emerging strategies for exploiting cannabinoid receptor agonists as medicines. Br J Pharmacol, 2009. 156 (3): p. 397-411.

35. Lanciego, J.L., et al., Expression of the $m R N A$ coding the cannabinoid receptor 2 in the pallidal complex of Macaca fascicularis.J Psychopharmacol, 2011. 25 (1): p. 97-104.

36. Sousa, O.V., et al., Antinociceptive and anti-inflammatory effects of the essential oil from Eremanthus erythropappus leaves. J Pharm Pharmacol, 2008. 60 (6): p. 771-7.

37. Mishra, D., et al., Chemical composition and analgesic activity of Senecio rufinervis essential oil. Pharm Biol, 2010.48 (11): p. 1297-301. 
38. Hernandez-Leon, A., et al., Role of beta-Caryophyllene in the Antinociceptive and Anti-Inflammatory Effects of Tagetes lucida Cav. Essential Oil. Molecules, 2020. 25 (3).

39. Menezes, I.A., et al., Antinociceptive effect and acute toxicity of the essential oil of Hyptis fruticosa in mice. Fitoterapia, 2007. 78 (3): p. 192-5.

40. Khalilzadeh, E., et al., Antinociceptive effects, acute toxicity and chemical composition of Vitex agnuscastus essential oil.Avicenna J Phytomed, 2015. 5 (3): p. 218-30.

41. Segat, G.C., et al., Antiallodynic effect of beta-caryophyllene on paclitaxel-induced peripheral neuropathy in mice.Neuropharmacology, 2017. 125 : p. 207-219.

42. Fiorenzani, P., et al., In vitro and in vivo characterization of the new analgesic combination Betacaryophyllene and docosahexaenoic Acid. Evid Based Complement Alternat Med, 2014. 2014 : p. 596312.

43. Finn, D.P., et al., Cannabinoids, the endocannabinoid system, and pain: a review of preclinical studies. Pain, 2021.

44. King, A.R., et al., URB602 inhibits monoacylglycerol lipase and selectively blocks 2-arachidonoylglycerol degradation in intact brain slices. Chem Biol, 2007. 14 (12): p. 1357-65.

45. Jung, K.M. and D. Piomelli, Assay of Monoacylglycerol Lipase Activity. Methods Mol Biol, 2016. 1412 : p. $157-68$.

46. Kinsey, S.G., et al., Repeated low-dose administration of the monoacylglycerol lipase inhibitor JZL184 retains cannabinoid receptor type 1-mediated antinociceptive and gastroprotective effects. J Pharmacol Exp Ther, 2013. 345 (3): p. 492-501.

47. Ignatowska-Jankowska, B., et al., Selective monoacylglycerol lipase inhibitors: antinociceptive versus cannabimimetic effects in mice. J Pharmacol Exp Ther, 2015. 353 (2): p. 424-32.

48. Long, J.Z., et al., Dual blockade of FAAH and MAGL identifies behavioral processes regulated by endocannabinoid crosstalk in vivo.Proc Natl Acad Sci U S A, 2009. 106 (48): p. 20270-5.

49. Sun, J., et al., The endocannabinoid system: Novel targets for treating cancer induced bone pain. Biomed Pharmacother, 2019.120 : p. 109504.

50. Baggelaar, M.P., M. Maccarrone, and M. van der Stelt,2-Arachidonoylglycerol: A signaling lipid with manifold actions in the brain. Prog Lipid Res, 2018. 71 : p. 1-17.

51. Varga, Z.V., et al., beta-Caryophyllene protects against alcoholic steatohepatitis by attenuating inflammation and metabolic dysregulation in mice. Br J Pharmacol, 2018. 175 (2): p. 320-334.

52. Liu, H., et al., Physicochemical characterization and pharmacokinetics evaluation of betacaryophyllene/beta-cyclodextrin inclusion complex. Int J Pharm, 2013. 450 (1-2): p. 304-10.

53. Lou, J., et al.,beta-Caryophyllene/Hydroxypropyl-beta-Cyclodextrin Inclusion Complex Improves Cognitive Deficits in Rats with Vascular Dementia through the Cannabinoid Receptor Type 2 -Mediated Pathway. Front Pharmacol, 2017. 8 : p. 2. 